Retraction

\title{
Retraction: Han, J. et al. The Influence of Texture on Co/SBA-15 Catalyst Performance for Fischer-Tropsch Synthesis. Catalysts 2018, 8, 661
}

\author{
Jun Han ${ }^{1,2}{ }^{(D}$, Zijiang Xiong ${ }^{1}$, Zelin Zhang ${ }^{1}$, Hongjie Zhang ${ }^{1, * \mathbb{D}}$, Peng Zhou ${ }^{3}$ and Fei Yu ${ }^{3, *} \mathbb{C}$ \\ 1 Hubei Key Laboratory for Efficient Utilization and Agglomeration of Metallurgic Mineral Resources, \\ Wuhan University of Science and Technology, Wuhan 430081, China; hanjun@wust.edu.cn (J.H.); \\ xzj0507@outlook.com (Z.X.); zhangzelin@wust.edu.cn (Z.Z.) \\ 2 Industrial Safety Engineering Technology Research Center of Hubei Province, Wuhan University of Science \\ and Technology, Wuhan 430081, China \\ 3 Department of Agricultural and Biological Engineering, Mississippi State University, \\ Mississippi State, MS 39762, USA; zhoupengwust@hotmail.com \\ * Correspondence: zhanghongjie@wust.edu.cn (H.Z.); fyu@abe.msstate.edu (F.Y.); Tel.: +86-27-6886-2880 \\ (H.Z.)
}

Received: 7 May 2019; Accepted: 9 May 2019; Published: 13 May 2019

We have been made aware that the figures and experimental data of this article [1] are duplicated from another publication by Lu et al. [2].

MDPI is a member of the Committee on Publication Ethics and takes the responsibility to enforce strict ethical policies and standards very seriously. To ensure the addition of only high-quality scientific works to the field of scholarly publication, the present paper [1] is retracted and shall be marked accordingly. The article is retracted with the agreement of all authors. We apologize to the readership of Catalysts for any inconvenience caused.

\section{References}

1. Han, J.; Xiong, Z.; Zhang, Z.; Zhang, H.; Zhou, P.; Yu, F. The influence of texture on Co/SBA-15 catalyst performance for Fischer-Tropsch synthesis. Catalysts 2018, 8, 661. [CrossRef]

2. Lu, Y.; Zhou, P.; Han, J.; Yu, F. Fischer-Tropsch synthesis of liquid hydrocarbon over mesoporous SBA-15 supported cobalt catalysts. RSC Adv. 2015, 5, 59792-59803. [CrossRef] 